\title{
Experimental study of quantum coherence decomposition and trade-off relations in a tripartite system
}

\author{
Zhe Ding ${ }^{1,2,12}$, Ran Liu ${ }^{1,2,12}$, Chandrashekar Radhakrishnan $\mathbb{D}^{3,4,12}$, Wenchao Ma ${ }^{1,2,11}$, Xinhua Peng (iD) ${ }^{1,2,5}$, Ya Wang (iD) \\ Tim Byrnes ${ }^{6,7,8,9,10 凶}$, Fazhan Shi (iD) ${ }^{1,2,5 凶}$ and Jiangfeng Du (iD ${ }^{1,2,5}$ '
}

Quantum coherence is the most fundamental of all quantum quantifiers, underlying other well-known quantities such as entanglement. It can be distributed in a multipartite system in various ways-for example, in a bipartite system it can exist within subsystems (local coherence) or collectively between the subsystems (global coherence), and exhibits a trade-off relation. In this paper, we experimentally verify these coherence trade-off relations in adiabatically evolved nuclear spin systems using an NMR spectrometer. We study the full set of coherence trade-off relations between the original state, the bipartite product state, the tripartite product state, and the decohered product state. We also experimentally verify the monogamy inequality and show that both the quantum systems are polygamous during the evolution. We find that the properties of the state in terms of coherence and monogamy are equivalent. This illustrates the utility of using coherence as a characterization tool for quantum states.

npj Quantum Information (2021)7:145; https://doi.org/10.1038/s41534-021-00485-0

\section{INTRODUCTION}

Quantum coherence has been the focus of investigation in numerous fields such as quantum optics where the fundamental nature of coherence has been investigated using phase-space distributions and higher order correlation functions ${ }^{1-3}$. It was quantified recently in a quantum-information theoretic way ${ }^{4}$ and the modern view is that it is the broadest quantum properties and is at the root of various quantum quantifiers such as discord, entanglement, EPR steering, and Bell correlations ${ }^{5,6}$. A set of axioms were formally introduced which need to be satisfied by a coherence quantifier ${ }^{4}$. This gave rise to the field of resource theories of quantum coherence ${ }^{7-10}$, along with an explosion of interest in the measurement of coherence ${ }^{11-15}$ and its applications $^{16-19}$

Quantum coherence has some unique features not present in other quantifiers such as entanglement and quantum discord (for a review see ref. ${ }^{9}$ ). One feature is that coherence is a basis dependent property and hence the amount of coherence depends upon the chosen measurement basis. Additionally, coherence can localize in a unipartite system as quantum superposition, or be present as correlations between different qubits $^{12}$. For example, in a bipartite entangled state $(|00\rangle+|11\rangle) / \sqrt{2}$, the coherence is delocalized and cannot be attributed to any particular qubit. On the other hand, in a separable state $|++\rangle=(|0\rangle+|1\rangle)(|0\rangle+|1\rangle) / 2$, the coherence is localized within the qubits. In fact, a maximally entangled state has only global coherence and no local coherence; meanwhile product states are the opposite. This example illustrates the presence of a trade-off between the local and the global coherence in a quantum system. This trade-off is the simplest case and highlights the different complementary distributions of coherence in two qubits. In a multipartite system, there are more possible distributions of coherence and hence other types of trade-off relations.

In this work, we use an adiabatically evolved spin system to experimentally measure coherence and investigate the trade-off relations in tripartite systems. The tripartite trade-off relations are an application of the theory presented in ref. ${ }^{12}$, where it was found that the total coherence could be decomposed into local and global coherence. We theoretically define three new inequality trade-off relations for the tripartite system which further characterize the quantum state. In the experiment, we consider two different classes of quantum systems with two and threebody interactions. The quantum coherence is measured at different stages of an adiabatic evolution and various trade-off relations are verified. An example of this process is shown in Fig. $1 \mathrm{a}$, where initially the coherence is completely localized within the qubits. When the system is adiabatically evolved, it has both local and global coherence. At the end of the adiabatic evolution, the system has only global coherence. In addition to verifying the trade-off relations and coherence distributions, we analyze the monogamy of coherence. Monogamy, first introduced in the context of entanglement ${ }^{20,21}$, implies that, when Alice and Bob are maximally entangled, they are impossible to be simultaneously entangled with a third party Charlie. This concept was later extended to quantum correlation ${ }^{22,23}$ and quantum coherence ${ }^{12}$. We show that using the various coherence quantifiers and monogamy, one can reveal that despite the apparent differences between the two Hamiltonians, the decompositions of the coherence are in fact similar. This illustrates the utility of our

\footnotetext{
'Hefei National Laboratory for Physical Sciences at the Microscale and Department of Modern Physics, University of Science and Technology of China, 230026 Hefei, China. ${ }^{2}$ CAS Key Laboratory of Microscale Magnetic Resonance, University of Science and Technology of China, 230026 Hefei, China. ${ }^{3}$ Centre for Quantum Information, Communication and Computing, Indian Institute of Technology Madras, Chennai 600036, India. ${ }^{4}$ Centre for Quantum Science and Technology, Chennai Institute of Technology, Chennai 600069, India. ${ }^{5}$ Synergetic Innovation Center of Quantum Information and Quantum Physics, University of Science and Technology of China, 230026 Hefei, China. ${ }^{6}$ New York University Shanghai, 1555 Century Ave, 200122 Shanghai, Pudong, China. ${ }^{7}$ State Key Laboratory of Precision Spectroscopy, School of Physical and Material Sciences, East China Normal University, 200062 Shanghai, China. ${ }^{8}$ NYU-ECNU Institute of Physics at NYU Shanghai, 3663 Zhongshan Road North, 200062 Shanghai, China. ${ }^{9}$ National Institute of Informatics, 2-1-2 Hitotsubashi Chiyoda-ku, Tokyo 101-8430, Japan. ${ }^{10}$ Department of Physics, New York University, New York, NY 10003, USA. ${ }^{11}$ Present address: Department of Chemistry, Massachusetts Institute of Technology, Cambridge, MA 02139, USA. ${ }^{12}$ These authors contributed equally: Zhe Ding, Ran Liu, Chandrashekar Radhakrishnan. ${ }^{\bowtie}$ email: tim.byrnes@nyu.edu; fzshi@ustc.edu.cn; djf@ustc.edu.cn
} 
a

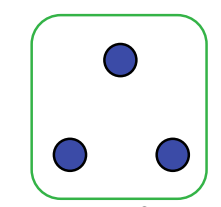

$t=0$

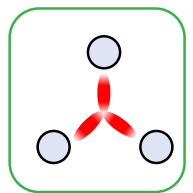

$t=2 T / 3$
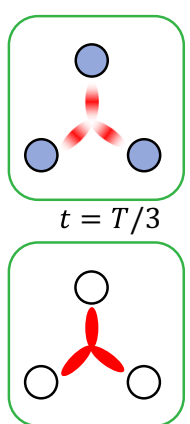

$t=T$ b

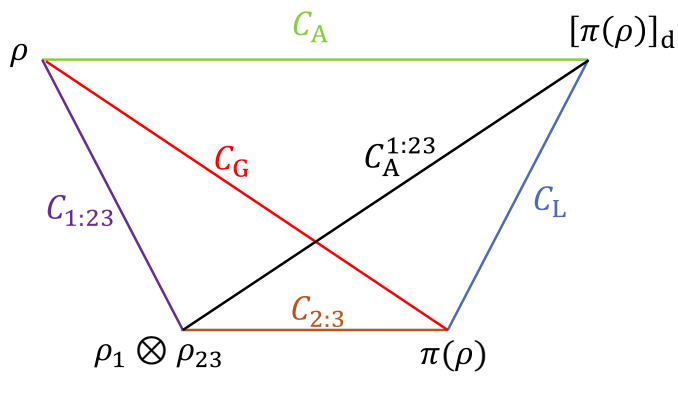

Fig. 1 Demonstration of the coherence distribution. a Quantum coherence trade-off is described in tripartite systems with circles representing the qubits. The blue discs and red leaves represent the local coherence $\mathcal{C}_{\mathrm{L}}$ and the global coherence $\mathcal{C}_{\mathrm{G}}$. The strength of the color indicates the strength of the coherence. $\mathbf{b}$ A geometric picture of different coherences. Coherences are shown as distances between two different density matrices. $\rho$ is the original density matrix; $\pi(\rho)$ and $[\pi(\rho)]_{d}$ are as described in (5); $\rho_{1}=\operatorname{tr}_{2,3} \rho$ and $\rho_{23}=\operatorname{tr}_{1} \rho$ are the reduced density metrices.

approach where coherence can be used to characterize a state to reveal hidden similarities between different systems.

We note that several other works have examined quantum coherence experimentally recently ${ }^{24-27}$. A coherence witness was introduced in ref. ${ }^{24}$ to detect the total coherence through a violation of Leggett-Garg type inequality ${ }^{28}$. Meanwhile the amount of coherence in a single photonic qubit was measured experimentally $^{25}$ using the robustness of coherence ${ }^{13}$. These works studied coherence in unipartite systems and did not analyze the coherence decompositions, their distribution and the consequent trade-off relations in multipartite systems. Such experimental demonstrations, and our work in this paper, show that quantum coherence can be an effective characterization tool for quantum states. In particular, using the coherence decomposition methods as introduced in this work and ref. ${ }^{12}$, one can obtain insights into the nature of the quantum state that may not be obvious from the examination of the density matrix.

\section{RESULTS}

\section{Models}

In this work, we study two different tripartite quantum systems. The first system is an Ising model described by the Hamiltonian

$H_{z z}(t)=\omega_{z} \sum_{i=1,2,3} S_{i}^{z}+\omega_{x} \sum_{i=1,2,3} S_{i}^{x}+2 J_{2}(t) \sum_{1 \leq i<j \leq 3} S_{i}^{z} S_{j}^{z}$,

where $S_{i}^{z / x}$ is the nuclear spin in the $z / x$-direction and $J_{2}$ represents the two-body interaction strength with $\omega_{z}=-2$ being the magnetic field in the longitudinal direction. A small transverse field $\omega_{x}=0.1$ is provided to lift the degeneracy of the ground state so that the adiabatic evolution is possible. Initially when we set $J_{2}=0$ and $\omega_{x} \ll \omega_{z}$, the ground state is nearly a separable state $|000\rangle$. The state is adiabatically evolved by increasing $J_{2}$ from 0 to $\left|\omega_{z}\right|$ in order to obtain a state close to $|W\rangle=(|001\rangle+|010\rangle+$ $|100\rangle) / \sqrt{3}$ at the end of the evolution ${ }^{29}$. The fidelity between the final ground state and $|W\rangle$ is 0.9978 while our experimental final state has a fidelity to $|W\rangle$ as high as 0.9578 .

The second quantum system we consider has the following form:

$H_{z z z}(t)=\omega_{x} \sum_{i=1,2,3} S_{i}^{x}+4 J_{3}(t) S_{1}^{z} S_{2}^{z} S_{3}^{z}$,

where $J_{3}$ is the three-body interaction strength which varies from 0 to 5 during the adiabatic evolution. The corresponding initial and final ground states are $|---\rangle$ and, in the sense of zero-order perturbation, $|G\rangle=(|001\rangle+|010\rangle+|100\rangle+|111\rangle) / 2$, respectively.
The fidelity between the final ground state and $|G\rangle$ is 0.9996 while our experimental final state has a fidelity to $|G\rangle$ as high as 0.9661 . The final state has both bipartite and tripartite coherences. For both the quantum systems, the coherence is measured at each stage of the evolution using quantum tomography methods.

\section{Quantifying coherence}

To measure coherence we use the square root of quantum version of the Jensen-Shannon divergence (QJSD) ${ }^{30-33}$

$\mathcal{D}(\rho, \sigma)=\sqrt{\frac{1}{2}\left[\mathcal{S}_{\mathrm{r}}(\rho \|(\rho+\sigma) / 2)+\mathcal{S}_{\mathrm{r}}(\sigma \|(\rho+\sigma) / 2)\right]}$.

Here $\rho$ and $\sigma$ are two density matrices of the same dimensionality and $\mathcal{S}_{\mathrm{r}}\left(\rho_{1} \| \rho_{2}\right)=\operatorname{tr} \rho_{1} \log \left(\rho_{1} / \rho_{2}\right)$ is the quantum relative entropy. Using this measure, the total coherence in the system is

$\mathcal{C}_{\mathrm{T}}(\rho) \equiv \mathcal{D}\left(\rho, \rho_{\mathrm{d}}\right)$,

where $\rho$ is the density matrix and $\rho_{\mathrm{d}}=\sum_{k}\langle k|\rho| k\rangle|k\rangle\langle k|$ is the diagonal density matrix with $|k\rangle$ representing the eigenstates of $S_{j}^{z}$. The global and local coherence are defined, respectively, as ${ }^{12}$

$\mathcal{C}_{\mathrm{G}}(\rho) \equiv \mathcal{D}(\rho, \pi(\rho)) ; \quad \mathcal{C}_{\mathrm{L}} \equiv \mathcal{D}\left(\pi(\rho),[\pi(\rho)]_{\mathrm{d}}\right)$.

Here $\pi(\rho) \equiv \otimes_{i} \rho_{i}$, where $\rho_{i}=\operatorname{tr}_{\forall j \neq i} \rho$ and footnotes $i, j$ are indices of subsystems. Subscript $d$ indicates the diagonal part of the density matrix in the $S^{z}$-basis. In terms of the coherence trade-off, the more relevant quantity is the absolute coherence defined as

$\mathcal{C}_{\mathrm{A}}(\rho) \equiv \mathcal{D}\left(\rho,[\pi(\rho)]_{\mathrm{d}}\right)$,

which is different from the total coherence $\mathcal{C}_{\mathrm{T}}$ and is the total amount of coherence in the product basis. The reference state [ $\pi$ $(\rho)]_{d}$ for absolute coherence contains neither coherence or correlations between the subsystems, while the reference state for total coherence $\rho_{\mathrm{d}}$ can potentially contain classical correlations. Since our measure $\mathcal{D}(\rho, \sigma)$ satisfies the triangle inequality for a multipartite system up to five qubits according to numerical studies $^{34}$, we have the trade-off relation (see Fig. 1b).

$\mathcal{C}_{\mathrm{A}} \leq \mathcal{C}_{\mathrm{L}}+\mathcal{C}_{\mathrm{G}}$.

The total coherence $\mathcal{C}_{\mathrm{T}}$ does not satisfy this trade-off relation since the reference state $\pi(\rho)$ is used.

One of the interesting aspects of tripartite systems is that coherence can be distributed in different ways. It is well-known that for entanglement, GHZ and $\mathrm{W}$ states are two different classes of tripartite entangled states ${ }^{35}$. The entanglement in a $\mathrm{GHZ}$ state is genuinely tripartite, whereas in a W state, the entanglement is bipartite in nature. In this context, it is interesting to examine the 

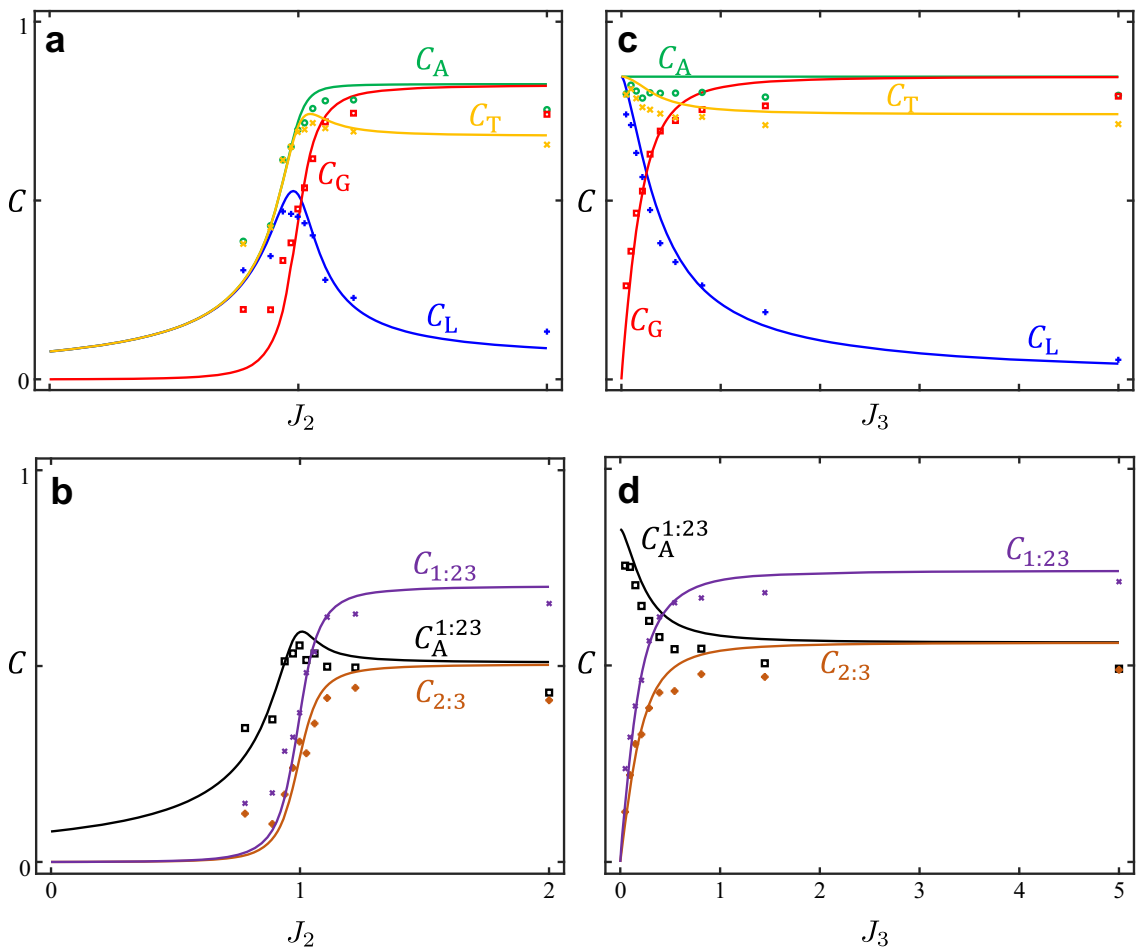

Fig. 2 The variation of different coherences as a function of the interaction parameters. Plot for the Hamiltonian $H_{z z}$ in $(\mathbf{a}, \mathbf{b})$ and $H_{z z z}$ in (c, d). The experimental data are shown by the points and the lines show the theoretically obtained results.

coherence additionally in a bipartite fashion. To this end, we introduce a geometrical picture as shown in Fig. $1 \mathrm{~b}$, by considering $\rho_{1} \otimes \rho_{23}$. We evaluate the coherence between qubit 1 and the bipartite block 23 according to

$\mathcal{C}_{1: 23} \equiv \mathcal{D}\left(\rho, \rho_{1} \otimes \rho_{23}\right), \quad \mathcal{C}_{2: 3} \equiv \mathcal{D}\left(\rho_{23}, \rho_{2} \otimes \rho_{3}\right)$.

Here $\mathcal{C}_{1: 23}$ measures the coherence between qubit 1 and the bipartite block 23 and $\mathcal{C}_{2: 3}$ is the coherence in the bipartite block 23. We note that including $\rho_{1}$ does not make any difference to the global coherence, and $\mathcal{C}_{2: 3}=\mathcal{D}\left(\rho_{1} \otimes \rho_{23}, \pi(\rho)\right)$; the proof is given in the Supplementary Note 1. Complementary to the coherence $\mathcal{C}_{1: 23}$ we have the contribution

$\mathcal{C}_{\mathrm{A}}^{1: 23} \equiv \mathcal{D}\left(\rho_{1} \otimes \rho_{23},[\pi(\rho)]_{\mathrm{d}}\right)$.

Based on these coherence distributions we have the following trade-off relations

$\mathcal{C}_{\mathrm{A}} \leq \mathcal{C}_{1: 23}+\mathcal{C}_{\mathrm{A}}^{1: 23} ; \quad \mathcal{C}_{\mathrm{A}}^{1: 23} \leq \mathcal{C}_{2: 3}+\mathcal{C}_{\mathrm{L}}$

Since $\mathcal{C}_{1: 23}$ and $\mathcal{C}_{2: 3}$ are global coherences, they give another trade-off relation

$\mathcal{C}_{\mathrm{G}} \leq \mathcal{C}_{1: 23}+\mathcal{C}_{2: 3}$.

The four equations in (7), (10) and (11) correspond to the four triangles that are present in Fig. 1b. They are also four trade-off relations that can be verified for the generated tripartite states.

\section{Coherence trade-off}

The variation of the coherence contributions during the evolution is shown in Fig. 2. There are mainly four types of errors leading to the deviation between experimental and theoretical results: initial state imperfection, decoherence, pulse error and measurement error. Please refer to Supplementary Note 4 for a detailed analysis. For Hamiltonian $H_{z z}$ at $J_{2}=0$, the interactions are turned off and the ground state is a product state. It is locally rotated from the state $|000\rangle\left(\mathcal{C}_{\mathrm{L}}=\mathcal{C}_{G}=0\right)$, due to the transverse field $\omega_{x}$ which induces a small local coherence. We observe that there are two regions, $J_{2} \in[0,1)$ with $\mathcal{C}_{\mathrm{L}}$ and $\mathcal{C}_{\mathrm{G}}$ increasing and $J_{2} \in[1,2]$ with $\mathcal{C}_{\mathrm{L}}$ decreasing and $\mathcal{C}_{\mathrm{G}}$ increasing. The crossover at $J_{2}=1$ corresponds to a quantum phase transition in a spin system with two-body interactions ${ }^{29}$. For the Hamiltonian $H_{z z z}$ at $J_{3}=0$, the ground state is $|---\rangle$, a coherent product state and hence $\mathcal{C}_{\mathrm{G}}=0$ and $\mathcal{C}_{\mathrm{L}}$ is maximal. At $J_{3}=5$, the ground state is nearly $|G\rangle$ for which $\mathcal{C}_{\mathrm{L}}=0$ and $\mathcal{C}_{G}$ is maximal. The two distinct regions of $H_{z z z}$ are $J_{3} \in[0,0.25)$ $\left(\mathcal{C}_{\mathrm{L}}>\mathcal{C}_{\mathrm{G}}\right)$ and $J_{3} \in[0.25,5]\left(\mathcal{C}_{\mathrm{L}}<\mathcal{C}_{\mathrm{G}}\right)$ with the crossover at $J_{3}=0.25$. They are related to a critical point at $J_{3}=\omega_{x}$ for a spin system with three-body interaction in the thermal dynamic limit ${ }^{29,36-41}$. We note that there are regions where $\mathcal{C}_{\mathrm{G}}>\mathcal{C}_{\mathrm{T}}$ for both $H_{\mathrm{zz}}$ and $H_{\mathrm{zzz}}$. This is due to our definition of global coherence, where all correlations between the qubits are broken by forming a product state, whereas in the definition of total coherence, classical correlations can be present in the decohered state. This verifies that $\mathcal{C}_{\mathrm{A}}$ is the more appropriate quantity in the context of trade-off relations.

To visualize the expected trade-off relations according to (4), (7) and (11), we look at the ratios $\mathcal{C}_{\mathrm{G}} / \mathcal{C}_{\mathrm{L}}, \mathcal{C}_{2: 3} / \mathcal{C}_{\mathrm{L}}, \mathcal{C}_{1: 23} / \mathcal{C}_{\mathrm{A}}^{1: 23}$ and $\mathcal{C}_{2: 3} / \mathcal{C}_{1: 23}$ using both experimental data and the corresponding theoretical results as shown in Fig. 3. We observe three types of trade-off behavior corresponding to complete, partial and no trade-off. For ratios of $\mathcal{C}_{\mathrm{G}}$ and $\mathcal{C}_{2: 3}$ to $\mathcal{C}_{\mathrm{L}}$, there is a complete tradeoff between these quantities, since there is a complete exchange from locally to collectively distributed coherence. Meanwhile the comparison of $\mathcal{C}_{2: 3}$ and $\mathcal{C}_{\mathrm{A}}^{1: 23}$ to $\mathcal{C}_{1: 23}$ only results in a partial tradeoff, where the ratios saturate to a finite value. In these cases, since both quantities in the ratio are types of global coherence, the ratios saturate to these particular values decided by large $J_{2}, J_{3}$. For Hamiltonian $H_{z z}$, the ratio $\mathcal{C}_{2: 3} / \mathcal{C}_{1: 23}$ remains constant throughout. We attribute this to the fact that for this Hamiltonian, there is a complete qubit symmetry, such that the entangled component of the state is always of the form of a $W$ state. Hence when comparing two types of correlation-type coherences, although the amount of coherence both become small as $J_{2} \rightarrow 0$, their ratio remains the same. 

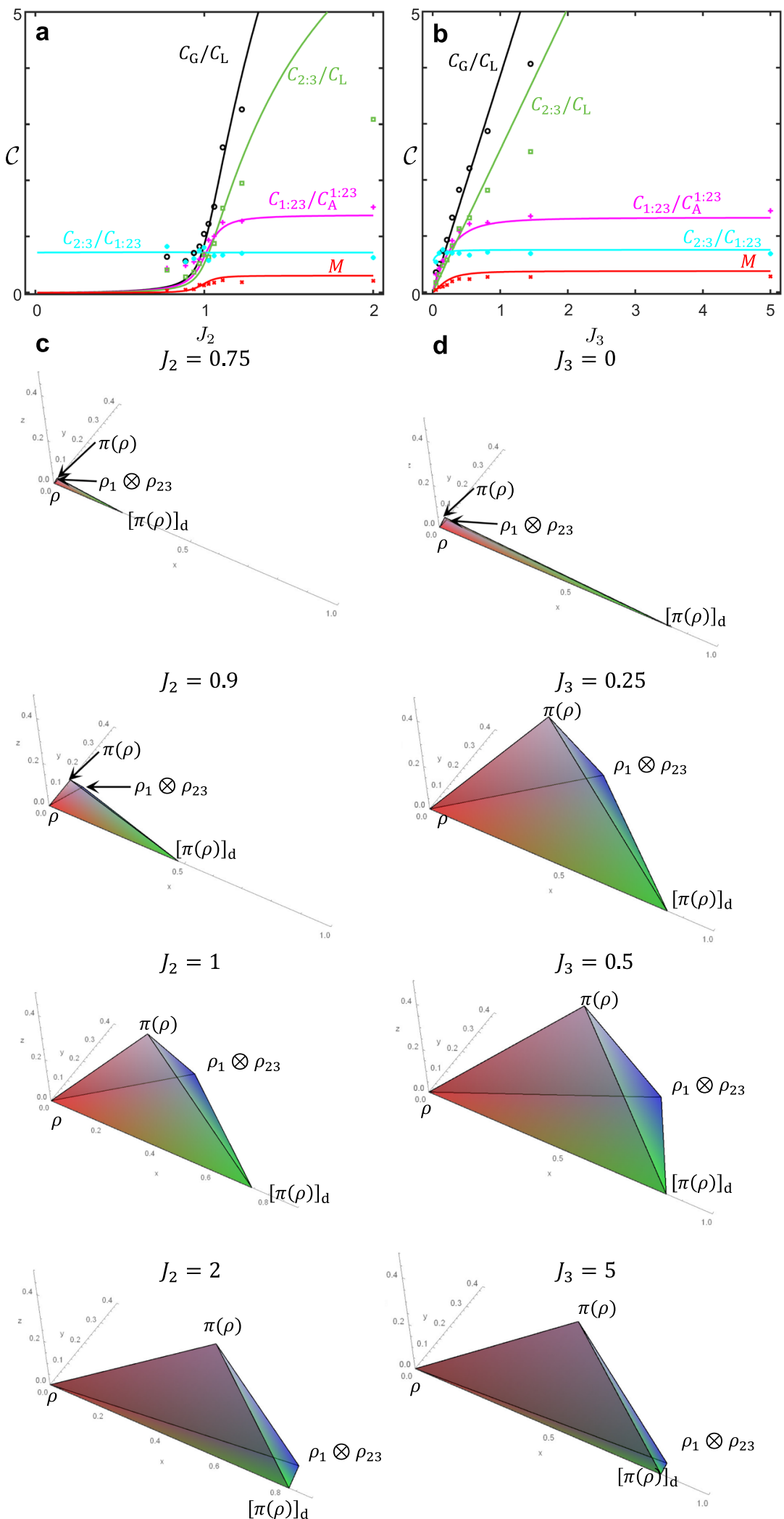

Fig. 3 Visualization of the trade-off relations. The ratio between the different pairs of coherences and monogamy of coherence is shown in a Hamiltonian $H_{z z}$ and $\mathbf{b}$ Hamiltonian $H_{z z z}$ as a function of interaction parameters. The points represent the experimental data and the solid lines correspond to the theoretical calculation. $\mathbf{c}, \mathbf{d}$ Geometric plots of the coherence in three-dimensional Euclidean space for the $H_{z z}$ and $H_{z z z}$ respectively, for the values of interaction parameters as marked (top to bottom). The lengths of the edges are taken to be the coherence contributions as shown in Fig. $1 \mathrm{~b}$. The coordinates of the state $\rho$ are $(0,0,0) ;[\pi(\rho)]_{\mathrm{d}}$ is $\left(\mathcal{C}_{\mathrm{A}}, 0,0\right) ; \pi(\rho)$ is $\left(\mathcal{C}_{\mathrm{G}} \cos \theta, \mathcal{C}_{\mathrm{G}} \sin \theta, 0\right) ; \rho_{1} \otimes \rho_{12}$ is $\left(\mathcal{C}_{1: 23} \cos \phi, \mathcal{C}_{1: 23} \sin \phi \cos \xi, \mathcal{C}_{1: 23} \sin \phi \sin \xi\right)$, where the angles are chosen to match the coherences as in Fig. $1 \mathrm{~b}$. 
a

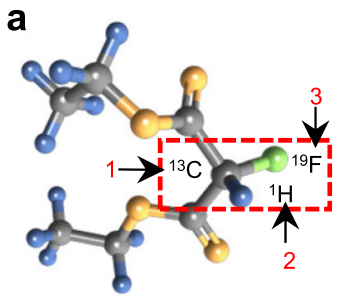

b

1

2

3

\begin{tabular}{llll}
\hline 1 & $100 \mathrm{MHz}$ & & \\
2 & $160.5 \mathrm{~Hz}$ & $400 \mathrm{MHz}$ & \\
3 & $-194.4 \mathrm{~Hz}$ & $47.9 \mathrm{~Hz}$ & $376 \mathrm{MHz}$
\end{tabular}

C

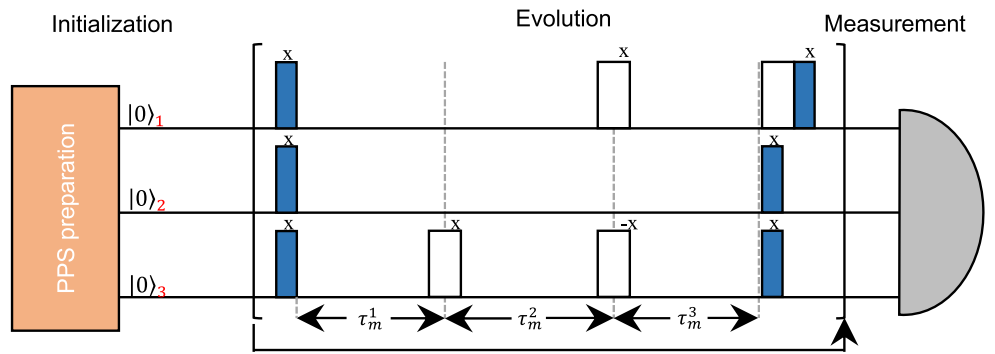

d

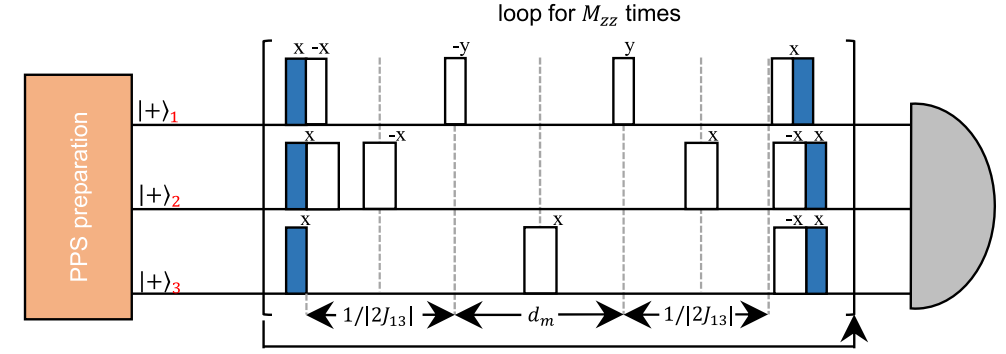

loop for $M_{z z Z}$ times

Fig. 4 Experimental setup. a Molecular structure of Diethyl fluoromalonate. The three nuclear spins ${ }^{13} \mathrm{C},{ }^{1} \mathrm{H}$ and ${ }^{19} \mathrm{~F}$ adopted in the experiment are labeled. The corresponding qubit index is also marked in red for each nuclear spin with a black arrow. $\mathbf{b}$ Parameters of the natural Hamiltonian of the three-spin system are shown in this table. The diagonal terms are the values of the chemical shift and the offdiagonal terms represent the scalar coupling between the different nuclei. $\mathbf{c}$ and $\mathbf{d}$ shows the schematic diagram explaining the experimental procedure for $H_{z z}$ and $H_{z z z}$, respectively. In the initialization part, we prepare the system from a pseudopure state (PPS) into the designed Hamiltonian's ground state. In the evolution part, a discrete refocusing scheme is used to perform two Hamiltonians. Combining with Trotter expansion, we can perform adiabatic evolution under any interaction. The wide and narrow unfilled pulses represent $\pi$ and $\pi / 2$ pulses, respectively while the rotation axes are labeled above each pulse. The filled pulses represent a rotation of $\omega_{x} \tau / 2$, where $\tau$ means the length of each Trotter slice. The measurement part is carried out using quantum state tomography.

The different kinds of coherences can also be visualized geometrically as shown in Fig. $3 c$, d. Here, we plot the various coherences by assigning them Euclidean distances in three dimensional space. From the results, we find that while $H_{z z}$ and $H_{z z z}$ have different kinds of interactions, their coherence distributions evolve similarly. The general behavior is that the states $\pi(\rho)$ and $\rho_{1} \otimes \rho_{12}$ start in the vicinity of $\rho$, then eventually move to a location near $[\pi(\rho)]_{d}$, along different trajectories. The primary difference between the two Hamiltonians is that $H_{z z z}$ always has a constant $\mathcal{C}_{A}$, hence the size of the tetrahedron is of the same order, whereas for $H_{z z}$ the tetrahedron starts from a point. However, apart from the overall magnitude of the coherence, the distribution of coherences are remarkably similar for both cases.

\section{Monogamy of coherence}

The monogamy of coherence describes the trade-off between the bipartite and tripartite global coherences of a three-body quantum system $^{12}$. We can quantify the monogamy according to $M=\mathcal{C}_{1: 2}+\mathcal{C}_{1: 3}-\mathcal{C}_{1: 23}$, where $M>0$ corresponds to a polygamous system and $M \leq 0$ to a monogamous system. The monogamy of coherence for the two Hamiltonians is shown in Fig. 3a, b. We find that the quantum systems are polygamous for every value of the interaction parameter except for the initial value $J_{2}, J_{3}=0$. This points to the fact that for both the quantum systems, the most dominant form of global coherence is the bipartite global coherence. Since the coherences $\mathcal{C}_{1: 23}$ and $\mathcal{C}_{2: 3}$ are global coherences, it is only natural that they are related to $\mathcal{C}_{G}$, the total global coherence as explained in (11). This confirms the picture provided by Fig. $3 c$, d, that the coherence generated in the two Hamiltonians is of the same type. This arises fundamentally because of the similar nature of the $|W\rangle$ and $|G\rangle$ state, which both have an bipartite-like entanglement structure.

\section{DISCUSSION}

We extended the notion of coherence trade-offs introduced in ref. ${ }^{12}$ and experimentally studied all the trade-offs that are possible with the four-point decompositions as shown in Fig. $1 \mathrm{~b}$. Each point in the diagram corresponds to removing a coherence contribution. For example, the state $\pi(\rho)$ removes all the interqubit coherence and the state $[\pi(\rho)]_{d}$ removes all the coherence including that lying within the qubits. Since we are dealing with a tripartite system, we further performed a bipartite decomposition where the coherence between site 1 and bipartite block 23 is removed. Our results point to the fact that the trade-off relations are generic behavior and are always obeyed as we move from a separable state to an entangled state. The trade-off behavior is also consistent with approaches where coherence is considered a resource, and coherence is converted into different forms ${ }^{42}$, which may have different sensitivities to decoherence ${ }^{19,43}$. We also 
examined the distribution of global coherence using the property of monogamy of coherence and it was found that both the states were polygamous except when the interactions were turned off. The characterization of a quantum system through the coherence distribution diagrams in Fig. $3 c$, d was shown to be an effective tool to visualize the quantum state.

It is interesting to note that while $H_{z z}$ and $H_{z z z}$ have different types of interactions and different initial states, the final quantum states have similar quantum properties. In fact, they are both highly entangled states and symmetric under spin permutations, which is not necessarily obvious by simply examining their wavefunctions. For instance, the final state of $H_{z z z}$ is $|G\rangle=(|001\rangle+|010\rangle+|100\rangle+|111\rangle) / 2$, which is not a wellknown entangled state in comparison to the $W$ state, the final state of $H_{z z}$. As a matter of fact, $|G\rangle$ is a result of a local Hadamard operation of a GHZ state, which is more entangled than a W state. By comparing the two final states' coherence contributions quantitatively, it is revealed that the global coherence of $H_{z z}$ is lower than that of $\mathrm{H}_{z z z}$. Hence, using the coherence decompositions and the trade-off relations, one can gain insights into the essential character of a given state, which may not be obvious simply by examining the wavefunction.

\section{METHODS}

\section{Setup}

We experimentally simulate $H_{z z}$ and $H_{z z z}$ with nuclear spin qubits in this work. Diethyl fluoromalonate molecules are used to perform the target adiabatic evolution via a Trotter decomposition on a $400 \mathrm{MHz}(9.4 \mathrm{~T}) \mathrm{NMR}$ spectrometer at $303 \mathrm{~K}$. The molecular structure of diethyl fluoromalonate is shown in Fig. 4a. The three nuclear spins ${ }^{13} \mathrm{C},{ }^{1} \mathrm{H}$ and ${ }^{19} \mathrm{~F}$ in the molecule acts as the qubits. The natural Hamiltonian of the system is

$$
H_{\text {spin }}=\sum_{i=1,2,3} 2 \pi \delta_{i} S_{i}^{z}+\sum_{1 \leq i \leq j \leq 3} 2 \pi J_{i j} S_{i}^{z} S_{j}^{z}
$$

where $\delta_{i}$ is the chemical shift of the nuclear spin and $J_{i j}$ is the coupling between the ith and the jth nucleus as given in Fig. $4 \mathrm{~b}$.

In the first step of the experiment, a pseudopure state (PPS) of the form $\rho=(1-\mu) I / 8+\mu|\psi\rangle\langle\psi|$ is prepared from thermal equilibrium state using a line-selective method ${ }^{44}$, where $|\psi\rangle$ is an arbitrary pure state. Here the mixing parameter $\mu \approx 10^{-5}$ and $I$ denotes the $8 \times 8$ identity matrix. The adiabatic pathway is numerically optimized to generate the desired ground state. The schematic diagram of the sequence to fulfill $H_{z z}$ and $H_{z z z}$ are shown in Fig. 4c, d. At each stage, the corresponding density matrices are reconstructed using tomographic techniques.

\section{Sequence design}

The adiabatic evolution is performed in discrete steps in the experiment ${ }^{29,45,46}$. We label Hamiltonians $H_{z z}$ and $H_{z z z}$ as $H_{k}$, where index $k \in\{z z$, zzz\}. Notice that $H_{k}$ can be decomposed into different basis, $H_{k}=H_{k}^{x}+H_{k}^{y}$, in which $H_{k}^{x}$ contains only $S_{i}^{x}$ and $H_{k}^{y}$ contains only $S_{i}^{y}$. The evolution of each segment $U_{k, \exp }\left(t_{m}\right)$, is a Trotter expansion of the ideal one $U_{k, \text { ide }}\left(t_{m}\right)$, which can be expressed as

$$
\begin{aligned}
U_{k, \text { ide }}\left(t_{m}\right) & =\mathrm{e}^{-\mathrm{i}\left[H_{k}^{\alpha}+H_{k}^{2}\left(t_{m}\right)\right] \tau_{k}} \\
& =\mathrm{e}^{-\mathrm{i} H_{k}^{\alpha} \tau_{k} / 2} \mathrm{e}^{-\mathrm{i} H_{k}^{2}\left(t_{m}\right) \tau_{k}} \mathrm{e}^{-\mathrm{i} H_{k}^{\alpha} \tau_{k} / 2}+O\left(\tau^{3}\right) \\
& =U_{k, \exp }\left(t_{m}\right)+O\left(\tau^{3}\right)
\end{aligned}
$$

where $\tau_{k}$ is the interval of each step and $m \in\left[0, M_{k}\right]$ is the index of each step. We use a refocusing scheme to achieve each step in our work. In this method, tuned pulses are applied during each Trotter slice, and the Hamiltonian in each short time period is accurately controlled.

For Hamiltonian (1), the quantum system is adiabatically evolved by tuning the two qubit interaction strength adiabatically over the range $[0,2]$. Experimentally, the adiabatic state transfer (ASP) is performed in discrete steps, such that $J_{2}(t)$ assumes discrete value $J_{2}\left(t_{m}\right)$ with $m=0, \ldots, M_{z z}$. At each time step, the evolution is generated using multipulse sequence $U_{z z, \exp }\left(t_{m}\right)$ using Trotter expansion formula as described in Eq. (13).
The resulting Hamiltonian is

$$
\begin{aligned}
H_{z z}^{x} & =\omega_{x} \sum_{i=1,2,3} S_{i}^{x}, H_{z z}^{z}\left(J_{2}\left(t_{m}\right)\right) \\
& =\omega_{z} \sum_{i=1,2,3} S_{i}^{z}+2 J_{2}\left(t_{m}\right) \sum_{1 \leq i \leq j \leq 3} S_{i}^{z} S_{j}^{z}
\end{aligned}
$$

A schematic description of the refocusing scheme is shown in Fig. $4 \mathrm{c}$ where the narrow unfilled rectangles denote $\pi / 2$ pulses, and the wide ones show $\pi$ pulses. By defining $d_{i j}=1 /\left(2_{i j}\right)$, the width of filled pulse in the figure are all $\omega_{x} \tau_{z z} / 2$ and the radio-frequency offsets for three channels are set as $F Q 1_{m}=$ $\omega_{z} /\left(4 J_{2}\left(t_{m}\right) d_{12}\right), F Q 2_{m}=\omega_{z} /\left(4 J_{2}\left(t_{m}\right)\left(d_{12}+d_{13}+d_{23}\right)\right)$ and $F Q 3_{m}=\omega_{z} /\left(4 J_{2}\left(t_{m}\right)\right.$ $\left.d_{23}\right)$, the delays are $\tau_{m}^{1}=\frac{J_{2}\left(t_{m}\right) \tau_{z z}}{\pi} \times\left(d_{12}+d_{23}\right), \tau_{m}^{2}=\frac{J_{2}\left(t_{m}\right) \tau_{z z}}{\pi} \times\left(d_{12}+d_{13}\right)$, and $\tau_{m}^{3}=\frac{J_{2}\left(t_{m}\right) \tau_{z 3}}{\pi} \times\left(d_{13}+d_{23}\right)$.

Next we consider the Hamiltonian of a tripartite quantum system with $J_{3}$ being the three-body interaction strength as shown in Eq. (2). The interaction parameter $J_{3}$ is tuned adiabatically in the range $[0,5]$. Again, we use a discrete refocusing scheme in which $J_{3}(t)$ is discretized into $t_{m \prime} m=$ $0, \ldots, M_{z z z}$. The schematic diagram is shown in Fig. $4 \mathrm{~d}$ in which the width of the filled pulse are all $\omega_{x} \tau_{z z z} / 2$ and the delay $d_{m}=\frac{J_{3}\left(t_{m}\right) \tau_{z z z}}{\pi} \times d_{12}$.

From above, one can see that the unit of studied quantities like $J_{2}, \omega_{z}, \tau_{k}$ always cancel out when they come into the parameters of the experiment. This means that the units of them do not matter in the experiment, only the relative relations between them matter. So, they are in arbitrary units and we need not mention the unit during discussion. We use 0.7 and 0.4 as the value of $\tau_{z z}$ and $\tau_{z z z}$ when we design the experimental sequences. Please refer to Supplementary Note 3 for the optimization details of the parameters.

\section{DATA AVAILABILITY}

Data are available from the authors on reasonable request.

\section{CODE AVAILABILITY}

The codes used for calculating coherence components and error analysis are written in MATLAB and available from the authors on reasonable request.

Received: 18 March 2021; Accepted: 8 September 2021; Published online: 01 October 2021

\section{REFERENCES}

1. Glauber, R. J. Coherent and incoherent states of the radiation field. Phys. Rev. 131, 2766 (1963).

2. Sudarshan, E. Equivalence of semiclassical and quantum mechanical descriptions of statistical light beams. Phys. Rev. Lett. 10, 277 (1963).

3. Scully, M. O. \& Zubairy, M. S. Quantum Optics (Cambridge University Press, 1999).

4. Baumgratz, T., Cramer, M. \& Plenio, M. Quantifying coherence. Phys. Rev. Lett. 113, 140401 (2014)

5. Ma, Z.-H. et al. Operational advantage of basis-independent quantum coherence. EPL 125, 50005 (2019).

6. Adesso, G., Bromley, T. R. \& Cianciaruso, M. Measures and applications of quantum correlations. J. Phys. A Math. Theor. 49, 473001 (2016).

7. Winter, A. \& Yang, D. Operational resource theory of coherence. Phys. Rev. Lett. 116, 120404 (2016).

8. Chitambar, E. \& Gour, G. Critical examination of incoherent operations and a physically consistent resource theory of quantum coherence. Phys. Rev. Lett. 117, 030401 (2016).

9. Streltsov, A., Adesso, G. \& Plenio, M. B. Colloquium: quantum coherence as a resource. Rev. Mod. Phys. 89, 041003 (2017).

10. Streltsov, A., Rana, S., Boes, P. \& Eisert, J. Structure of the resource theory of quantum coherence. Phys. Rev. Lett. 119, 140402 (2017).

11. Shao, L.-H., Xi, Z., Fan, H. \& Li, Y. Fidelity and trace-norm distances for quantifying coherence. Phys. Rev. A 91, 042120 (2015).

12. Radhakrishnan, C., Parthasarathy, M., Jambulingam, S. \& Byrnes, T. Distribution of quantum coherence in multipartite systems. Phys. Rev. Lett. 116, 150504 (2016).

13. Napoli, C. et al. Robustness of coherence: an operational and observable measure of quantum coherence. Phys. Rev. Lett. 116, 150502 (2016).

14. Girolami, D. Observable measure of quantum coherence in finite dimensional systems. Phys. Rev. Lett. 113, 170401 (2014).

15. Radhakrishnan, C., Ding, Z., Shi, F., Du, J. \& Byrnes, T. Basis-independent quantum coherence and its distribution. Ann. Phys. 409, 167906 (2019). 
16. Streltsov, A. et al. Entanglement and coherence in quantum state merging. Phys. Rev. Lett. 116, 240405 (2016).

17. Karpat, G., Çakmak, B. \& Fanchini, F. Quantum coherence and uncertainty in the anisotropic xy chain. Phys. Rev. B 90, 104431 (2014).

18. Radhakrishnan, C., Ermakov, I. \& Byrnes, T. Quantum coherence of planar spin models with dzyaloshinsky-moriya interaction. Phys. Rev. A 96, 012341 (2017).

19. Radhakrishnan, C., Parthasarathy, M., Jambulingam, S. \& Byrnes, T. Quantum coherence of the heisenberg spin models with dzyaloshinsky-moriya interactions. Sci. Rep. 7, 13865 (2017).

20. Coffman, V., Kundu, J. \& Wootters, W. K. Distributed entanglement. Phys. Rev. A 61, 052306 (2000).

21. Koashi, M. \& Winter, A. Monogamy of quantum entanglement and other correlations. Phys. Rev. A 69, 022309 (2004).

22. Giorgi, G. L. Monogamy properties of quantum and classical correlations. Phys. Rev. A 84, 054301 (2011).

23. Prabhu, R., Pati, A. K., Sen, A. \& Sen, U. et al. Conditions for monogamy of quantum correlations: Greenberger-horne-zeilinger versus w states. Phys. Rev. A 85, 040102 (2012).

24. Zhou, Z.-Q., Huelga, S. F., Li, C.-F. \& Guo, G.-C. Experimental detection of quantum coherent evolution through the violation of Leggett-Garg-type inequalities. Phys. Rev. Lett. 115, 113002 (2015).

25. Wang, Y.-T. et al. Directly measuring the degree of quantum coherence using interference fringes. Phys. Rev. Lett. 118, 020403 (2017)

26. Wu, K.-D. et al. Quantum coherence and state conversion: theory and experiment npj Quantum Information 6, 1-9 (2020).

27. Yuan, Y. et al. Direct estimation of quantum coherence by collective measurements. npj Quantum Information 6, 1-5 (2020).

28. Leggett, A. J. Realism and the physical world. Rep. Prog. Phys. 71, 022001 (2008).

29. Peng, X., Zhang, J., Du, J. \& Suter, D. Ground-state entanglement in a system with many-body interactions. Phys. Rev. A 81, 042327 (2010).

30. Lin, J. Divergence measures based on the shannon entropy. IEEE Trans. Inf. Theory 37, 145-151 (1991)

31. Briët, J. \& Harremoës, P. Properties of classical and quantum Jensen-Shannon divergence. Phys. Rev. A 79, 052311 (2009).

32. Majtey, A., Lamberti, P. \& Prato, D. Jensen-Shannon divergence as a measure of distinguishability between mixed quantum states. Phys. Rev. A 72, 052310 (2005).

33. Lamberti, P., Majtey, A., Borras, A., Casas, M. \& Plastino, A. Metric character of the quantum Jensen-Shannon divergence. Phys. Rev. A 77, 052311 (2008).

34. Lamberti, P. W., Majtey, A. P., Borras, A., Casas, M. \& Plastino, A. Metric character of the quantum Jensen-Shannon divergence. Phys. Rev. A 77, 052311 (2008).

35. Dür, W., Vidal, G. \& Cirac, J. I. Three qubits can be entangled in two inequivalent ways. Phys. Rev. A 62, 062314 (2000).

36. Igloi, F. Conformal invariance and surface critical behaviour of a quantum chain with three-spin interaction. J. Phys. A: Math. Gen. 20, 5319-5324 (1987)

37. Penson, K. A., Debierre, J. M. \& Turban, L. Conformal invariance and critical behavior of a quantum Hamiltonian with three-spin coupling in a longitudinal field. Phys. Rev. B 37, 7884-7887 (1988).

38. Penson, K. A., Jullien, R. \& Pfeuty, P. Phase transitions in systems with multispin interactions. Phys. Rev. B 26, 6334-6337 (1982)

39. Igloi, F., Kapor, D. V., Skrinjar, M. \& Solyom, J. The critical behaviour of a quantum spin problem with thee-spin coupling. J. Phys. A: Math. Gen. 16, 4067-4071 (1983).

40. Baxter, R. J. \& Wu, F. Y. Exact solution of an ising model with three-spin interactions on a triangular lattice. Phys. Rev. Lett. 31, 1294-1297 (1973).

41. Pachos, J. K. \& Plenio, M. B. Three-spin interactions in optical lattices and criticality in cluster hamiltonians. Phys. Rev. Lett. 93, 056402 (2004).

42. Wu, K.-D. et al. Experimental cyclic interconversion between coherence and quantum correlations. Phys. Rev. Lett. 121, 050401 (2018).

43. $\mathrm{CaO}, \mathrm{H}$. et al. Fragility of quantum correlations and coherence in a multipartite photonic system. Phys. Rev. A 102, 012403 (2020).

44. Peng, X. et al. Preparation of pseudo-pure states by line-selective pulses in nuclear magnetic resonance. Chem. Phys. Lett. 340, 509-516 (2001).

45. Mitra, A., Ghosh, A., Das, R., Patel, A. \& Kumar, A. Experimental implementation of local adiabatic evolution algorithms by an NMR quantum information processor. J. Magn. Reson. 177, 285-298 (2005).
46. Peng, X., Zhang, J., Du, J. \& Suter, D. Quantum simulation of a system with competing two- and three-body interactions. Phys. Rev. Lett. 103, 140501 (2009).

\section{ACKNOWLEDGEMENTS}

The researchers at USTC are supported by the National Key Research and Development Program of China (Grants No. 2018 YFA0306600 and 2016YFA0502400), the National Natural Science Foundation of China (Grants Nos. $81788101,91636217,11722544,11761131011$, and 31971156), the CAS (Grants Nos. GJJSTD20200001, QYZDY-SSW-SLH004, and YIPA 2015370), the Anhui Initiative in Quantum Information Technologies (Grant No. AHY050000), the National Youth Talent Support Program. T.B. and R.C. are supported by the National Natural Science Foundation of China (62071301); State Council of the People's Republic of China (D1210036A); NSFC Research Fund for International Young Scientists (11850410426); NYU-ECNU Institute of Physics at NYU Shanghai; the Science and Technology Commission of Shanghai Municipality (19XD1423000); the China Science and Technology Exchange Center (NGA-16-001); the NYU Shanghai Boost Fund. R.C. was supported in part by a seed grant from IIT Madras to the Centre for Quantum Information, Communication and Computing.

\section{AUTHOR CONTRIBUTIONS}

J.D., F.S. and T.B. supervised the project. Z.D., R.L., C.R. and W.M. designed the experiments through discussion with X.P., Y.W., T.B., F.S. and J.D. R.L. and Z.D. performed the experiments, Z.D. and C.R. performed the calculations. Z.D., C.R., R.L., T.B. and F.S. wrote the paper. All authors analyzed the data, discussed the results, and agreed with the conclusions.

\section{COMPETING INTERESTS}

The authors declare no competing interests.

\section{ADDITIONAL INFORMATION}

Supplementary information The online version contains supplementary material available at https://doi.org/10.1038/s41534-021-00485-0.

Correspondence and requests for materials should be addressed to Tim Byrnes, Fazhan Shi or Jiangfeng Du.

Reprints and permission information is available at http://www.nature.com/ reprints

Publisher's note Springer Nature remains neutral with regard to jurisdictional claims in published maps and institutional affiliations.

\begin{abstract}
Open Access This article is licensed under a Creative Commons Attribution 4.0 International License, which permits use, sharing, daptation, distribution and reproduction in any medium or format, as long as you give appropriate credit to the original author(s) and the source, provide a link to the Creative Commons license, and indicate if changes were made. The images or other third party material in this article are included in the article's Creative Commons license, unless indicated otherwise in a credit line to the material. If material is not included in the article's Creative Commons license and your intended use is not permitted by statutory regulation or exceeds the permitted use, you will need to obtain permission directly from the copyright holder. To view a copy of this license, visit http://creativecommons. org/licenses/by/4.0/.
\end{abstract}

(c) The Author(s) 2021 